\title{
Die Briefe von Jacques-Louis Reverdin und Theodor Kocher an Anton v. Eiselsberg
}

Eine Quellenstudie zur Entdeckung der Ausfallserscheinungen bei Totalexstirpation der Schilddrüse

Von Markwart Michler und Jost Benedum

In seiner sorgfältigen Monographie Zur Geschichte der Schilddrüsen- und Kropfforschung im 19.Jahrhundert hat S.Bornhauser auch dem Prioritätsstreit zwischen Jacques Louis Reverdin und Theodor Kocher einen ausführlichen Abschnitt gewidmet ${ }^{1}$. Seine scharfsinnige Untersuchung über die Entdeckung der Ausfallserscheinungen bei der Totalexstirpation der Glandula thyreoidea führte ihn zu der Schlußfolgerung, daß Reverdin als erster den Zusammenhang zwischen der totalen Schilddrüsenentfernung und dem postoperativen kretinoiden Zustandsbild klar erkannte und auch als erster darüber berichtete; unbeschadet der Tatsache, daß Kocher die erste eingehende Schilderung dieser Folgezustände gegeben hat ${ }^{2}$. Dieser heute wohl allgemein anerkannten Feststellung läßt sich nichts Neues hinzufügen, sie bedarf keiner zusätzlichen Begründung.

Indessen stützte sich Bornhauser in seinen Ausführungen nicht allein auf die Publikationen der beiden Chirurgen, sondern auch auf den Briefwechsel, den sie in dieser Streitfrage mit Adolphe Combe und Anton v. Eiselsberg führten. Während aber Combe in seiner Arbeit über das Myxödem Kochers Brief mit nur geringen Auslassungen und den Reverdins offensichtlich vollständig veröffentlichte ${ }^{3}$, begnügte sich $v$. Eiselsberg in seiner Monographie über «Die Krankheiten der Schilddrüse» mit einer längeren Anmerkung ${ }^{4}$.

1 S. Bonnhauser, Zur Geschichte der Schilddrüsen- und Kropfforschung im 19. Jahrhundert (unter besonderer Berücksichtigung der Schweiz), Veröffentlichungen der Schweizerischen Gesellschaft für Geschichte der Medizin und der Naturwissenschaften XIX, Aarau 1951, S. $74 \mathrm{ff}$.

2 Bornhauser, S. 98.

3 A. Сомве, Le myxœdème, Rev. méd. Suisse rom. 17 (1897) $77 \mathrm{ff}$.

4 A.Freiherr v. Eiselsberg, Die Krankheiten der Schilddrüse, Deutsche Chirurgie 38, Stuttgart 1901, S.149f., Anm. 1. 
Auf dieses Resumé blieb auch Bornhauser in seiner Untersuchung angewiesen, da ihm die Briefe selbst damals nicht zugänglich waren. Sie befinden sich unterdessen im Besitz der Ludwig-Schunk-Bibliothek der Gießener Medizinischen Fakultät, und ihr Inhalt dürfte eine wertvolle Ergänzung unserer Kenntnisse über den Streit der beiden Gelehrten bilden. Dabei handelt es sich um zwei Briefe von Jacques Louis Reverdin und ebenfalls um zwei von Theodor Kocher.

Reverdins erster Brief an v. Eiselsberg ist ein Handschreiben, das mit dem Datum vom 25. November 1889 versehen ist, er bezieht sich daher noch nicht auf dessen später geplante Monographie über die Schilddrüsenerkrankungen. Der Inhalt läßt vielmehr erkennen, daß dieser ein Separatum von Reverdins Vortrag auf dem französischen Kongreß für Chirurgie vom 18. Oktober 1886 erbeten hatte ${ }^{5}$, und der Brief ist als Beischreiben zu dieser Sendung gedacht. Aus freien Stücken fügte Reverdin auch seine und seines Vetters Auguste gemeinsame Arbeit «Note sur vingt-deux opérations de goitre» bei ${ }^{6}$, « weil es für die Frage der historischen Darstellung des Myxoedems von Interesse ist ».

Sein zweiter Brief - mit Datum vom 8. Juni 1894 - ist fast fünf Jahre später verfaßt, er besteht aus einem kurzen handschriftlichen Anschreiben und einem maschinengeschriebenen Text von insgesamt vier Seiten. Zwar erwähnt hier Reverdin eingangs v. Eiselsbergs geplante Monographie, aber der tiefere Anlaß zu einem so ausführlichen Schreiben dürfte doch in der Publikation von Edmond Lardy und in Kochers Bemerkungen in der Deutschen Zeitschrift für Chirurgie zu suchen sein ${ }^{7}$. Zu seiner Verteidigung legt er zwei Sonderdrucke als Beweismittel bei, und nach seinen Ausführungen läßt sich voraussetzen, daß es sich bei dem einen um seine Mitteilung über die Folgen der totalen Entfernung der Schilddrüse handelte, deren Bedeutung von Bornhauser bereits entsprechend gewürdigt worden ist ${ }^{8}$. Überprüft man Reverdins Bibliographie und berücksichtigt, daß er nach eigener Aussage von den Aufsätzen «Conférence sur l'extirpation du goitre, faite à la clinique de M. le prof. Léon Tripier» und «Apropos de l'énucléation du

5 Vgl. J.L.Reverdin, Contribution à l'étude du myxœdème consécutif à l'extirpation totale ou partielle du corps thyroide, Congr. franç. chir.2, Paris 1886, und Rev. méd. Suisse rom. 7 (1887) 275. - Zum Inhalt vgl. BORNHAUSER, S. 144.

6 Erschienen in: Rev. méd. Suisse rom. 3 (1883) $169 \mathrm{ff}$.

7 E.LARdy, Contribution à l'histoire de la cachexie thyréoprive, Bern 1894, und Th. KoCHER, Zur Verhütung des Cretinismus und cretinoider Zustände nach neuen Forschungen, Dtsch. Z. Chir. 34. (1892) 580 f.

8 Vgl. Rev. méd. Suisse rom. 2 (1882) 539 und Bornhauser, S. $56 \mathrm{ff}$. und S. $84 \mathrm{ff}$. 
goitre » selbst keine Sonderdrucke mehr besaß, und daß er andererseits seinen Kongreßbericht «Contribution à l'étude du myxœdème consécutif à l'extirpation totale ou partielle du corps thyroide» bereits 1889 übersandt hatte, dann müßte das zweite Separatum die schon genannte, mit Auguste verfaßte Kasuistik betreffen, die er in seinem ersten Brief zunächst zurückerbeten hatte ${ }^{9}$.

Seine Hinweise auf Kochers «observation de 1874» und dessen «Arbeit von 1883 » beziehen sich auf die Abhandlung «Zur Pathologie und Therapie des Kropfes ${ }^{10}$ und auf den berühmten Bericht des Berner Chirurgen auf dem 12. Kongreß der Deutschen Gesellschaft für Chirurgie am 4. April 1883 in Berlin ${ }^{11}$, der die erbitterte Fehde einleitete. Das Zitat aus der Arbeit seines Vetters Auguste schließlich stammt aus dessen Aufsatz «De l'énucléation dans le traitement du goitre ${ }^{12}$, in dem dieser in scharfer Form gegen Kochers Behauptungen Stellung nahm.

Kochers erstes Schreiben datiert vom 15. Dezember 1894 und besteht aus zwei beidseitig beschriebenen Briefkarten. Er beruft sich ebenfalls auf v. Eiselsbergs geplante Monographie, und die wissenschaftlichen Publikationen, die er erwähnt, sind bereits in Reverdins Briefen zu Wort gekommen. Auch sein Vorwurf gegen den Vetter Auguste kann sich nur auf die nämlichen Auslassungen beziehen, die schon Jacques Louis Reverdin in seinem zweiten Brief anführt, und die von Bornhauser noch ausführlicher zitiert werden ${ }^{13}$.

Der zweite Brief des Berner Chirurgen - ein Handschreiben von vier Seiten in einem Quart-Format - ist im Ton noch drängender gehalten als der erste, ohne indessen neue Argumente beizubringen. Sein Hinweis auf Carl A. Ewald bezieht sich schließlich auf dessen damals kurz vor dem Abschluß stehende Monographie Die Erkrankungen der Schilddrüse, die ein Jahr später erschien.

Beide Briefschreiber erwähnen außerdem den englischen Laryngologen Felix Semon und den Bericht des englischen Myxoedemkomitees, das am 14. Dezember 1883 von der Clinical Society of London eingesetzt worden

9 Siehe Anm. 6.

10 Erschienen in: Dtsch. Z. Chir.4 (1874) $433 \mathrm{f}$.

11 Erschienen unter dem Titel: U̇ber Kropfexstirpation und ihre Folgen, Arch. klin. Chir.29 (1883) $254 \mathrm{ff}$.

12 Erschienen in: Rev. de chir. 3 (1892) $185 \mathrm{f}$.

13 Vgl. Bornhauser, S. $93 \mathrm{f}$. 
war, um die Beziehungen zwischen der Cachexia strumipriva und dem Myxoedem aufzuklären ${ }^{14}$.

Leider sind mit den Briefen weder die Anschriften noch die Couverts erhalten, und so läßt sich nur vermuten, daß Reverdins erster Brief nach Wien, sein zweiter und die Briefe Kochers aber nach Utrecht adressiert waren, da v. Eiselsberg in jenen Jahren den dortigen chirurgischen Lehrstuhl innehatte ${ }^{15}$. Auch geht aus keinem der vier Briefe hervor, ob dieser geantwortet und mit seinen beiden Schweizer Kollegen eine briefliche Diskussion über die Prioritätsfrage geführt hat. Entsprechende Konzepte oder gar Briefabschriften haben sich in der hiesigen Sammlung jedenfalls nicht angefunden.

Soweit die äußeren Daten und die Quellenliteratur, auf die sich die Briefe beziehen. Bevor jedoch noch kurz auf ihren Inhalt eingegangen wird, sollen sie im vollen Wortlaut vorgelegt werden, wobei orthographische Eigenheiten stehengelassen wurden.

\section{Die Briefe J.-L.Reverdins}

Très honoré Confrère

Genève 25 Nov. 89

Je vous adresse le travail que vous m'indiquez; la communication a éte faite au Congrès français de Chirurgie de 1886 (18 Octobre), le tirage à part que je vous envoie est extrait des compte rendus du Congres parus en 1887; le même travail a été imprimé dans le $\mathrm{N}^{\circ}$ de Juin de la Revue médicale de la Suisse Romande. Je vous prie de garder ce travail.

J'aurais voulu vous faire aussi hommage de l'autre travail: «Note sur vingt-deux opérations de goitre », mais je n'en possède plus qu'un exemplaire et vous serai obligé de me le renvoyer. Je vous l'envoie parcequ'il présente pour la question de l'historique du myxœdème de l'intérêt. Ce travail a paru dans les $\mathrm{N}^{\text {os }}$ de Avril Mai et Juin de la Revue Médicale de la Suisse Romande.

Veuillez présenter à Monsieur le Prof. Billroth mes souvenirs respectueux et agréer mes meilleures salutations.

Dr. J.-L. Reverdin

14 Näheres siehe: Bornhauser, S. $73 \mathrm{f}$. und S.114f.

15 Vgl. A.v. Eiselsberg, Lebensweg eines Chirurgen, Innsbruck/Wien 1938, S.106ff. - Zumindest für Reverdins ersten Brief wird man auf Grund der beigefügten Grüße an Billroth Wien als Empfangsort sicher annehmen dürfen. 


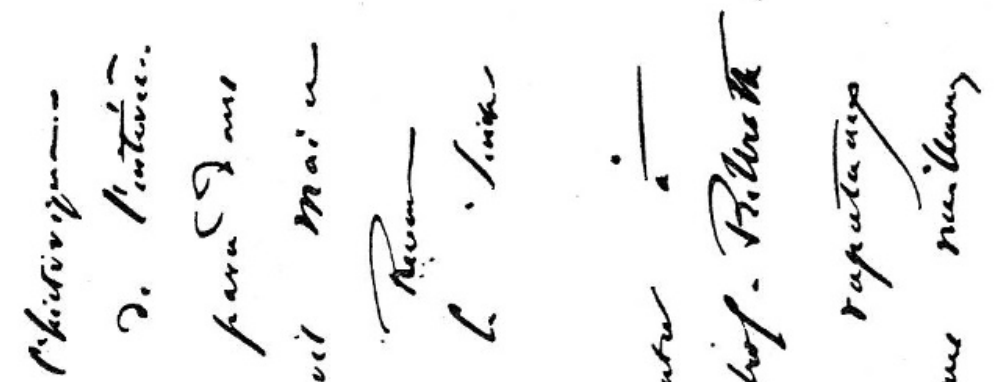

$$
\begin{aligned}
& \therefore \text {. } 3 x=1,0 \\
& \text { औ. }
\end{aligned}
$$

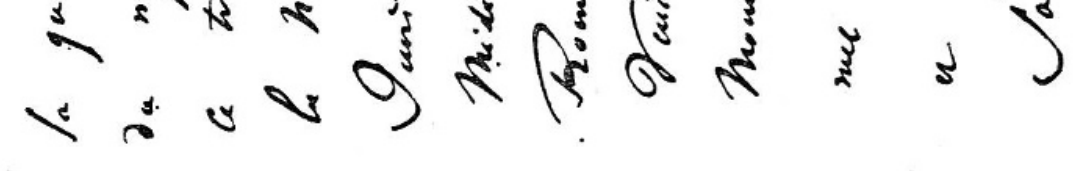
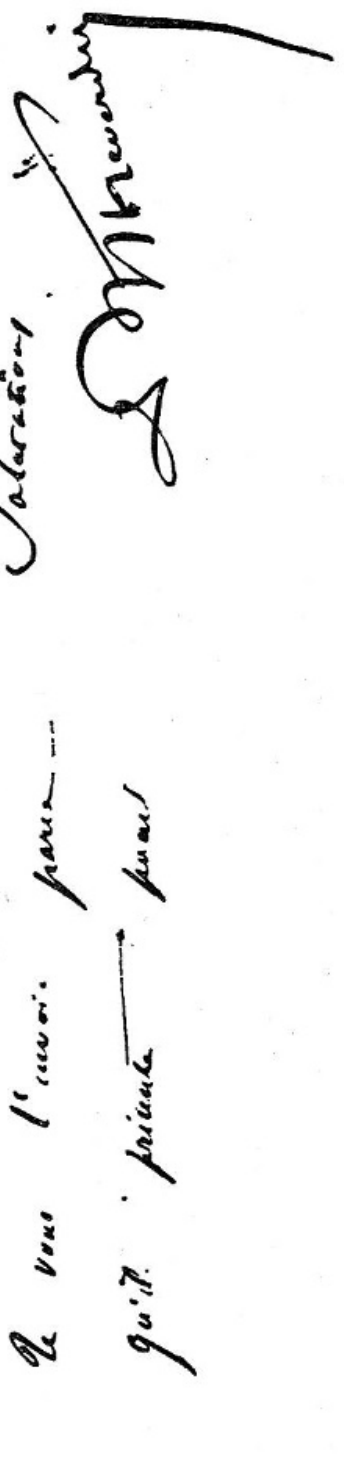

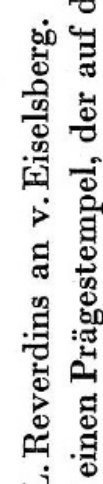

艼范

ค 졀

苍泀

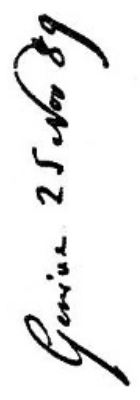

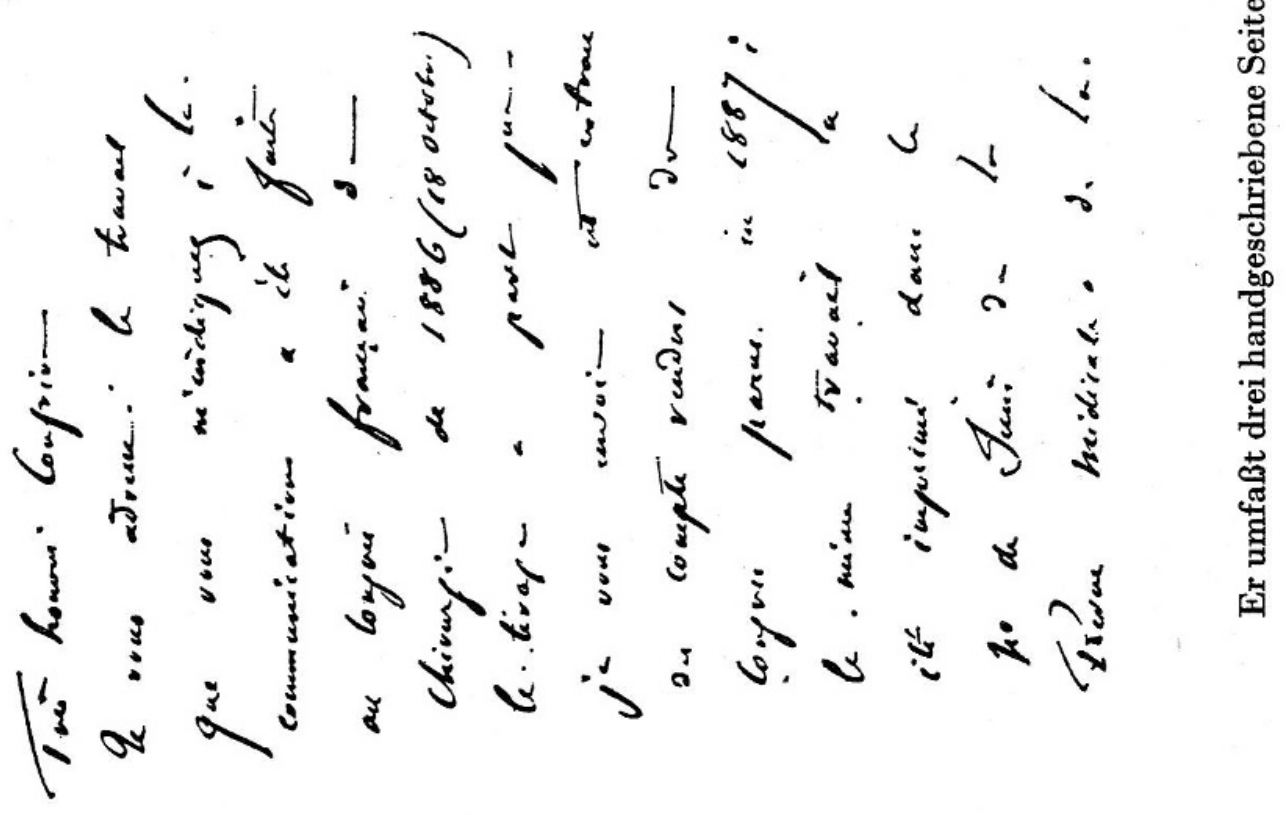


Très honoré collègue

Genève 8Juin 94

Je vous adresse ci-joint une lettre à la machine et je vous prie de m'excuser de ce fait (.) Mais il m'est difficile d'écrire lisiblement; excusez aussi les fautes dues au manque d'habitude.

J'espère que vous me pardonnerez de vous occuper d'une question si personnelle; Veuillez agréer très honoré collègue mes salutations empressées.

Dr.J.L. Reverdin

Prof. Jaques L. Reverdin

43 R. du Rhône

y'oncive 8 qum 94

Genève

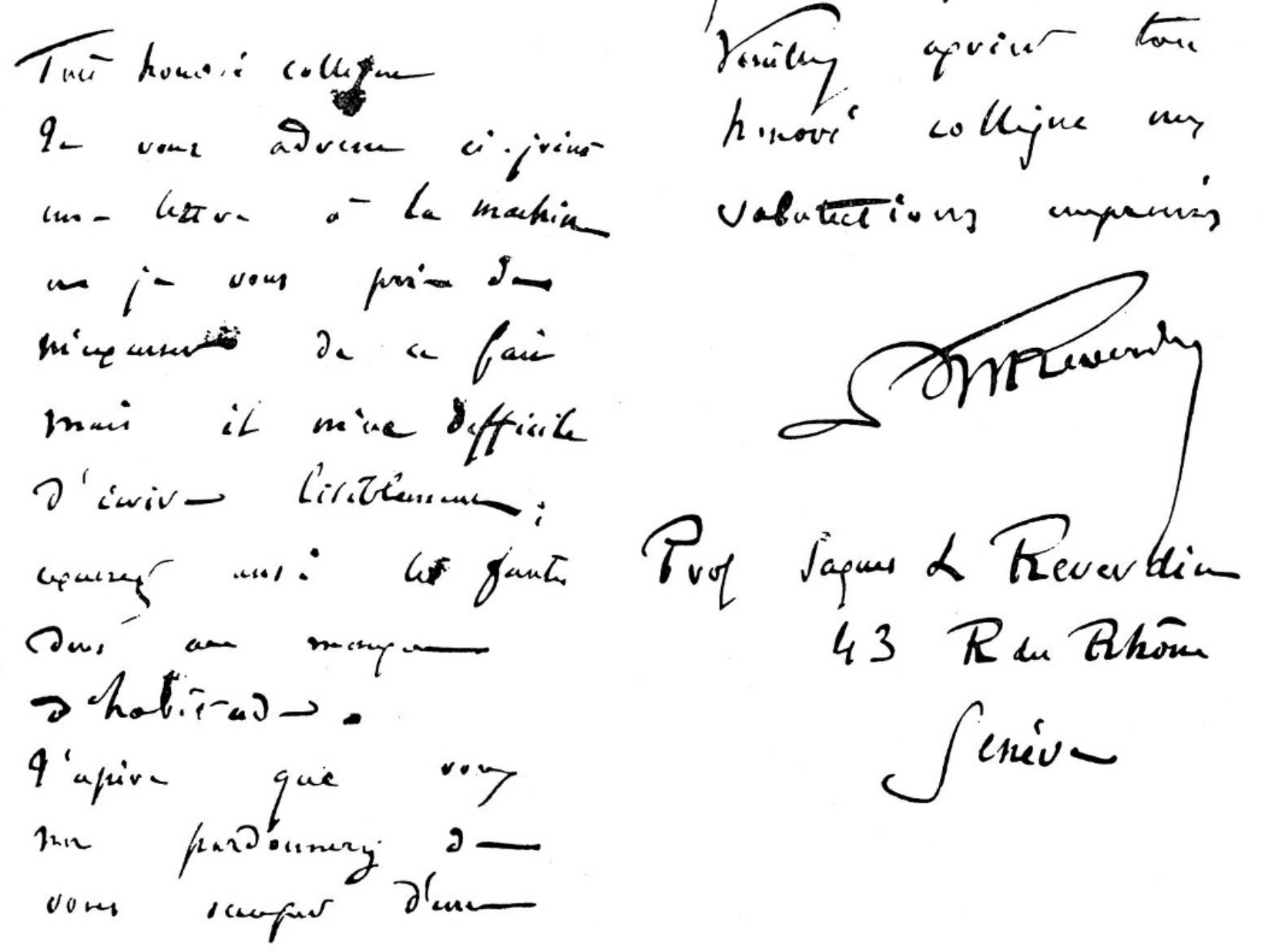

Zweiter Brief J.L. Reverdins an v.Eiselsberg. Das handschriftliche Begleitschreiben zu dem Brief in Maschinenschrift, auf dessen Faksimilewiedergabe verzichtet würde und der in der Folge abgedruckt ist 
Très honoré collégue

J'ai appris dernièrement que vous étiez chargé d'écrire pour la deutsche Chirurgie le volume traitant des maladies de la thyroide. Vous savez peut être que Monsieur le professeur Kocher me conteste d'avoir le premier signalé d'une façon formelle les accidents consécutifs à l'extirpation totale $[,]^{*}$ qu'il appelle cachexie tyréoprive[,] et que avec mon cousin A. Reverdin nous avons proposé de nommer myxoedème opératoire. Derni[è]rement j'ai eu connaissance par un de mes collégues d'une brochure signée Docteur Edmond Lardy et destinée à démontrer que je n'ai fait que piller Monsieur Kocher; monsieur Kocher, dans le $34^{\text {me }}$ volume de la deutsche Zeitschrift [f. Chirurgie] (pages 580 et 581) cherche à établir la même légende.

Je me permets don [c] de vous adresser deux tirages à part de me[s] publications sur le goitre; il faudrait y ajouter une leçon faite à Lyon à la clinique de mon regretté ami Léon Tripier, [pa] rue dans le Lyon médical en 1886 et un article sur l'énucléatio[n] dans la Semaine médicale; malheureusement je n'en ai plus un seul exemplaire.

Monsieur Kocher et Monsieur Lardy cherchent à établir que lors de la conversation que $\mathrm{j}$ 'eus avec mon collégue de Berne et où je lui demandai s'il avait vu des faits semblables à ceux que je lui signalais comme suite de l'extirpation totale, il faisait déja des recherches dans cette direction et que la communication faite la semaine suivante à la société de médecine de Genève sur ce sujet, l'a été pour lui enlever une découverte qui lui appartient ou au moins qui ne m'appartient pas.

Or c'est moi qui me suis adressé à Monsieur Kocher, qui lui ai dit sommairement ce que j'avais vu et lui ai demandé si[,] lui qui avait opéré beaucoup de goitres[,] avait vu chose semblable; il m'a répondu que non, que cependant il avait su qu'une de ses opérées avait présenté après son opération des altérations remarquables de la santé et du caractère.

Il est je crois très faci[le] de montrer qu'à cette époque le idées de Monsieur le professeur Kocher sur ce sujet devaient être dans un état embryonnaire si toutefois leur conception était un fait acompli et que son observation de 1874 malgré ses 8 ans de date n'avait pas porté de fruits.

Prenons en effet son travail de 1883 et nous verrons que ce chirurgie[n] ne reculait pas devant les extirpations totales de la [th] yroide.

Je trouve en effet dans sa communi[c] ation au congrès de Berlin de 1883

* Die Klammern [ ] bezeichnen handschriftliche Verbesserungen und Ergänzungen. 
indiquées 34 extirpati[o]ns totales, la première datant du 8.1.1874, les 30 [su] ivantes faites avant notre conversation et 3 depuis. Sur les 30 faites de Janvier 74 au 24.7.1882 il y en a 14 de janvier à juille[t] 1882, 5 en Juin, 2 en Juillet. De cette date au 2 Octobre, sauf un goît[re] malin indiqué comme opéré en automne, pas d'opérations, probablement à cause des vacances. Mais le 27.II. 82 Kocher extirpe un goitre folic[u]laire droit chez une malade dont le lobe gauche a déja été extirpé; il pratique l'extirpation totale encore le 27.II. 82 et le 16.I.83. Je remarque en outre [que] 24 fois il indique la date à laquelle le malade a été revu et le cachexie constatée et que c'est toujours dans les mois de Février et Mars 1883, aucune autre date d'ex[a]men d[e] malades au point de vue de la cachexie n'est indiquée; pour la malade de 1874 voici ce que je lis: [«] 9. Marie Bichsel II J.Struma follicularis aus multiplen Knollen. Starkes Wachsthum. Opérirt 8. I. 74. Medianschnit[t] sonst wie No.I. Total[e]xcision. Heilung in 4 Wochen. Cachexia strumipriva hohen Grades. Febr. 83.[»]

Voila pour la première acusation; il me semble que la démonstration est suffisante.

La seconde accusation est à l'état d'insinuation de la part de Monsieur Kocher[,] plus afirmative chez Monsieur Lardy.

Le travail que nous avons fait mon cousin et moi a paru dans mon Journal, la [R] evu[e] médicale de la Suisse romande dans les numéros du 15 Avril, 15 Mai et 15 Juin 1883; d'après Monsieur Lar[d] y tout ce qui a paru après le $21 \mathrm{Mai}$, jour auquel j'ai écrit à Monsieur Koch[e]r pour réclamer mes droits de priorité que j'estimais lésés dans son mémoire, doit être considéré comme ayant été fait d'après Kocher, les o[b] servations inspirées par son travail [et] tout cela il cherche à le démontrer au moyen de phrases séparées de leur contexte. Or dans les deux premières parties de notre travail nous avons annoncé que nous décririons certains faits nouveaux, que nous donnerions des appendices à quelques unes de nos observations; nous avons intercalé lorsque nous avons connu d'après les compte rendus des journeaux le travail de Kocher la phrase suivante:[«] Nous ne pouvons ni ne voulon[s] attendre la public[a]tion du travail de Kocher pour terminer cette étude; si nos vues concor[d] ent elles ne seront que plus affermies par le fait que nos recherches se sont faites d'une façon indépendante[»] etc. $\mathrm{J}[\mathrm{e}]$ vous envoie du reste un tirage à part où $\mathrm{j}$ 'a[i] souligné ce[r] tains passages propres à vous édifier.

Avant de terminer cette trop longue lettre je tiens à dire qu'en $\mathrm{c}[\mathrm{e}]$ qui me concerne je n'approuve pas compléte[m] ent cette phrase de mon cousin 
A. Reverdin dans la Revue de chirurgie:[«] Si j'insiste sur ce fait, c'est qu'il a son importance; car plus tard en Avril 1883, au congrès des chirurgiens allemands, le professeur Kocher de Berne présenta sous le titre de cachexie strumiprive l'ensemble de symptomes déja reconnus, décrits et publiés.[»]

Pour moi je prétends seulement avoir signalé le premier certains accidents consécutifs à l'extirpation totale d'une façon formelle. J'ajoute que dans le travail publié avec mon cousin en Avril, Mai et Juin 1883 nou avons les premiers montré la similitude de ces accidents avec le myxoedème spontané; Monsieur Semon qui a découvert plus tard ce rapport ne connaissait pas notre travail, comme il me l'a écrit du reste et comme cela est indiqué dans le rapport anglais sur le myxoedème.

Je suis bien loin de prétendre avoir du premier coup to[ut] vu et tout élucidé mais je pense cependant [avoir] contribué [pe]rsonnellement à l'étude de cette question intéressante.

Je vous fais toutes mes excuses, très honoré collégue, de vous adresser une si longue épitre mais vous comprendrez que je tienne à rétablir les faits devant des accusations qui s'attaquent a mon honorabilité scientifique.

Veuillez donc m'excuser et agréer mes salutations confraternelles.

Dr.J.L.Reverdin

Zu dem Brief auf den folgenden zwei Seiten:

Erster Brief Kochers an v. Eiselsberg. Er besteht aus zwei Briefkarten. Der Text der Rückseite der ersten Karte wird auf den Randzeilen der Rückseite fortgesetzt, springt dann auf die Randzeilen der Vorderseite zurück und geht dann erst auf die zweite Karte über. 


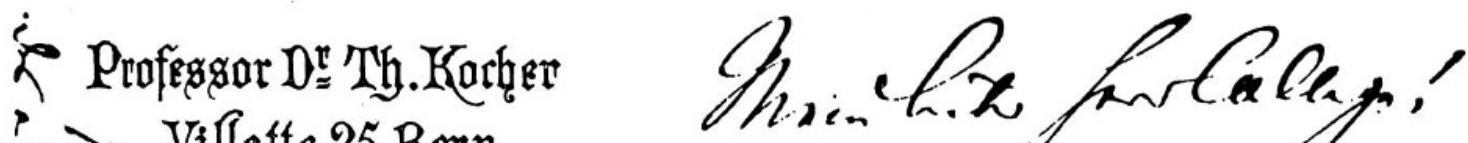
Xillette 25 Bern.

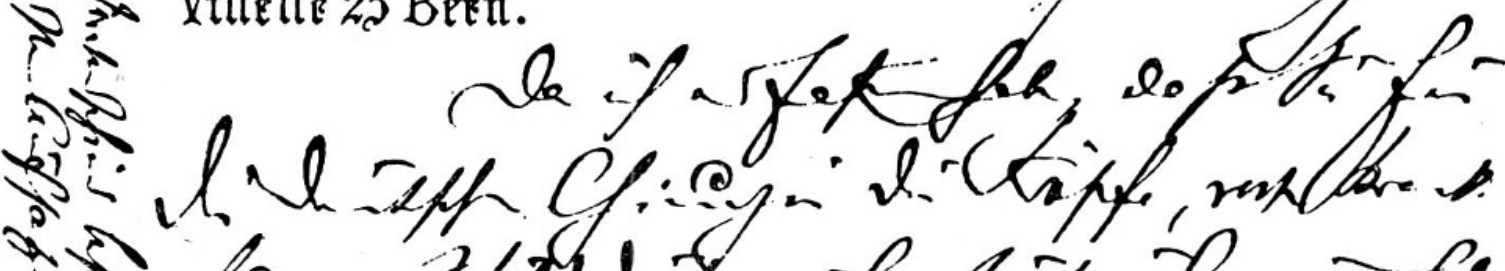

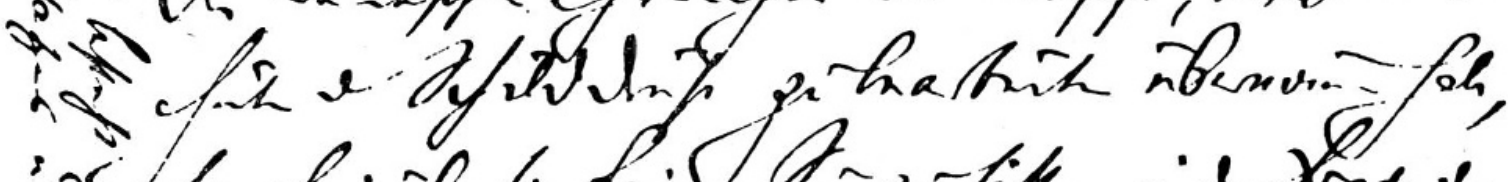

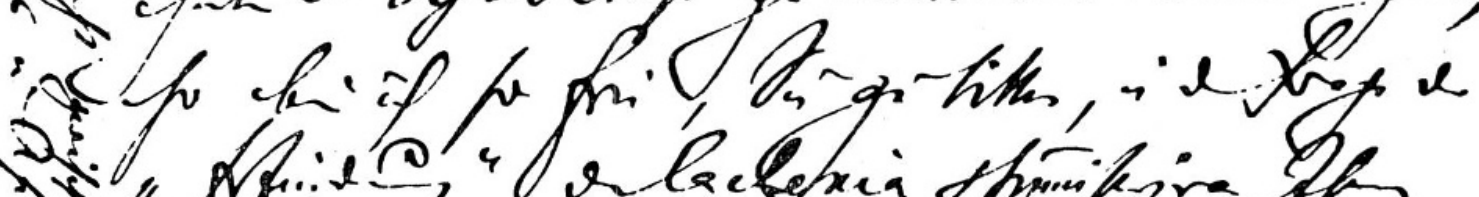

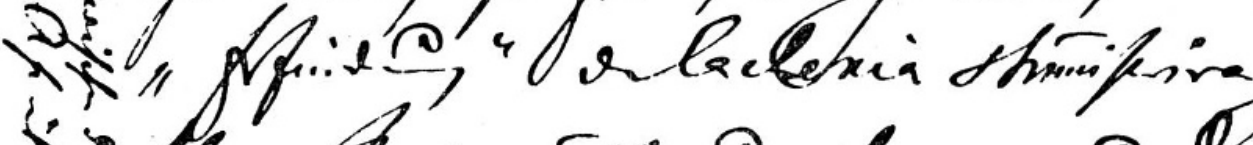

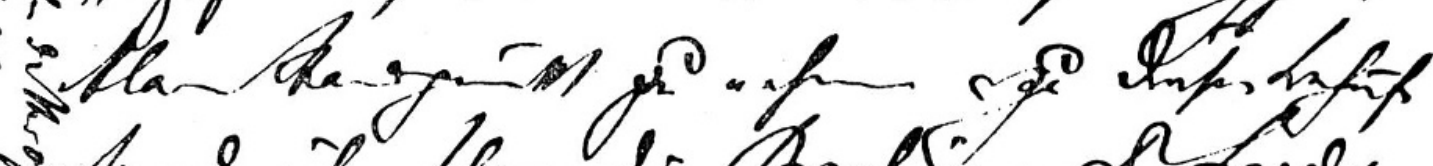

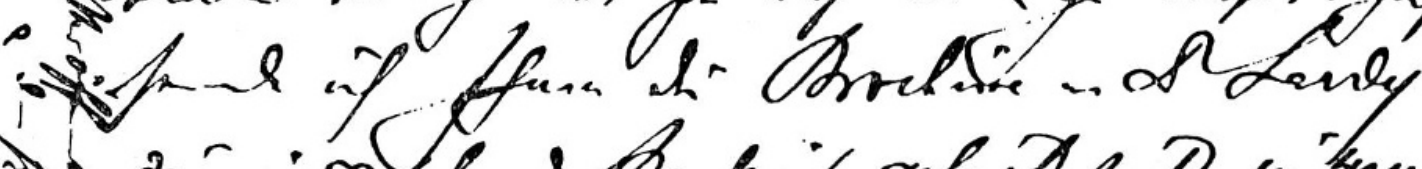

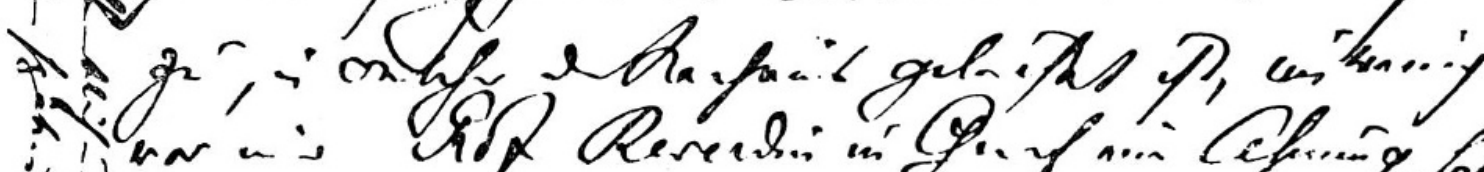

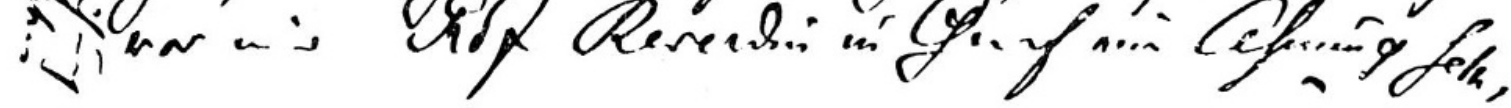

Erste Karte - Vorderseite

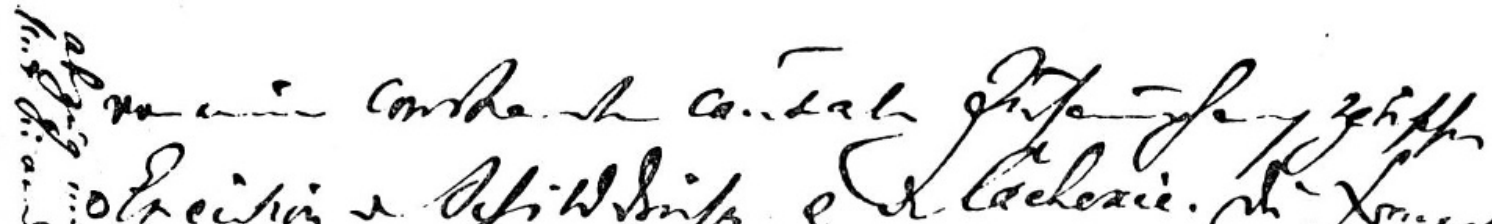

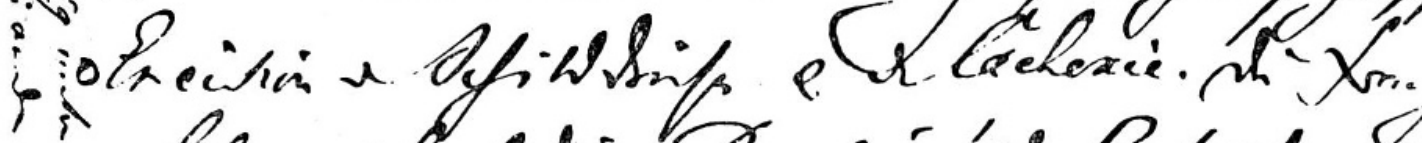

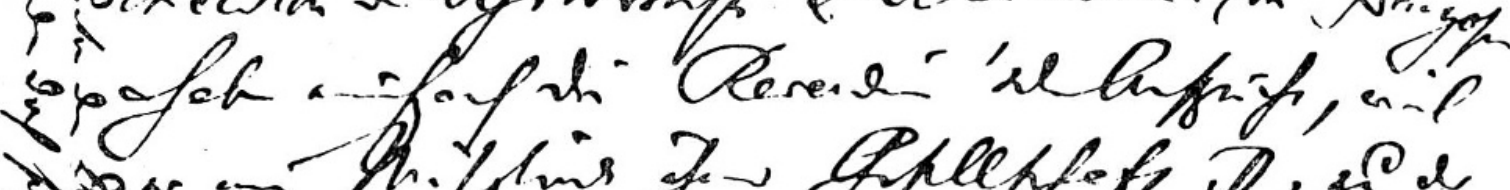

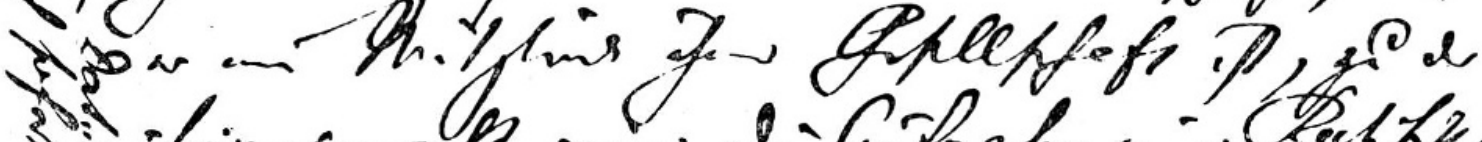

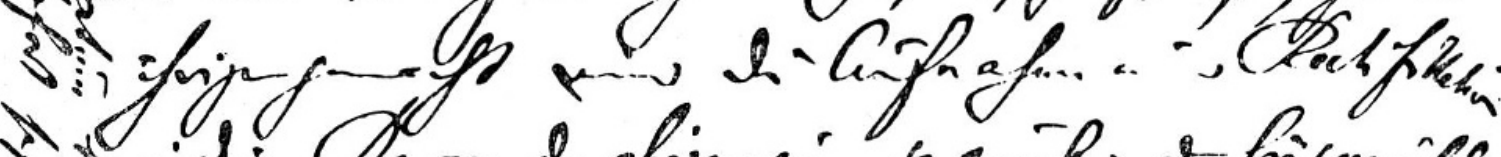

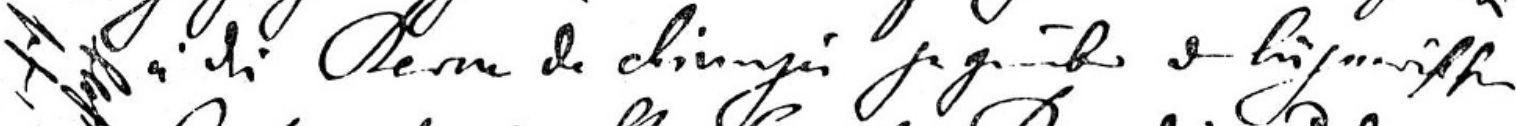

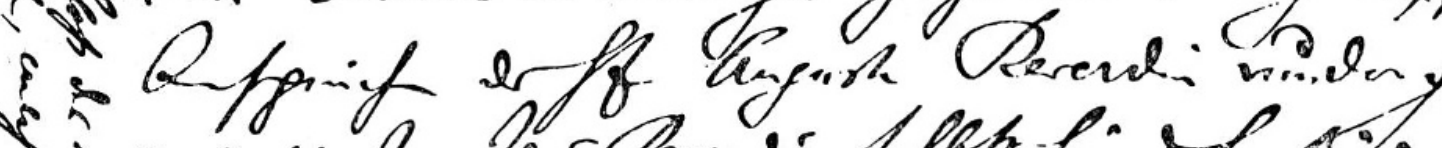

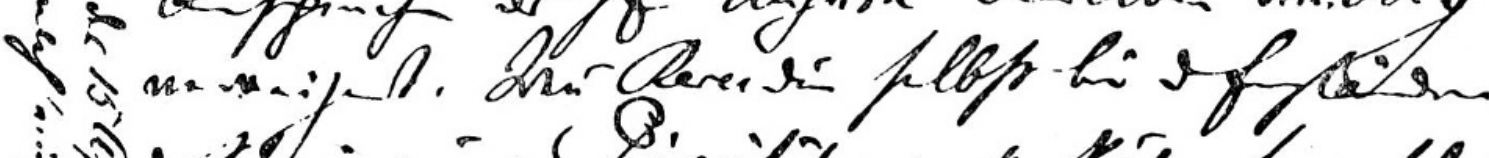

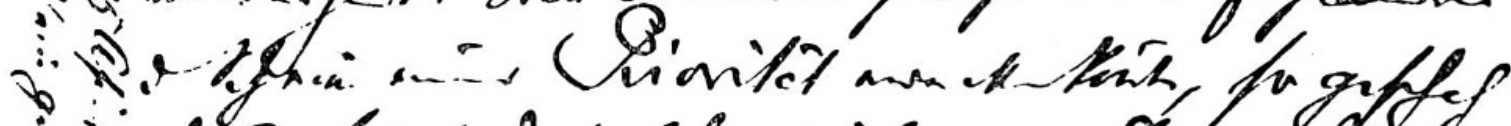

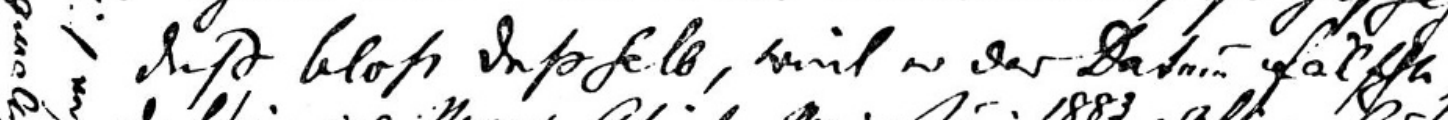

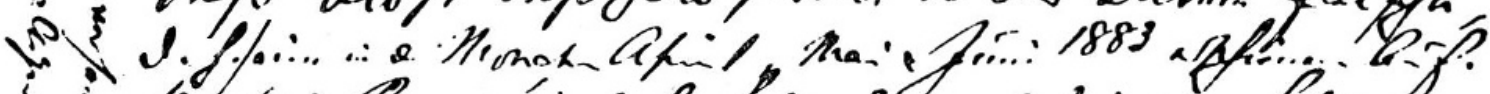

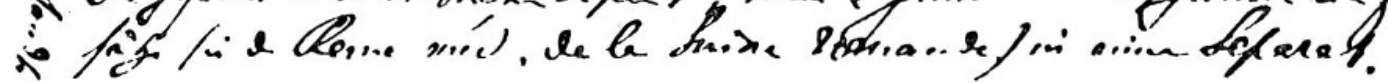

Erste Karte - Rückseite 


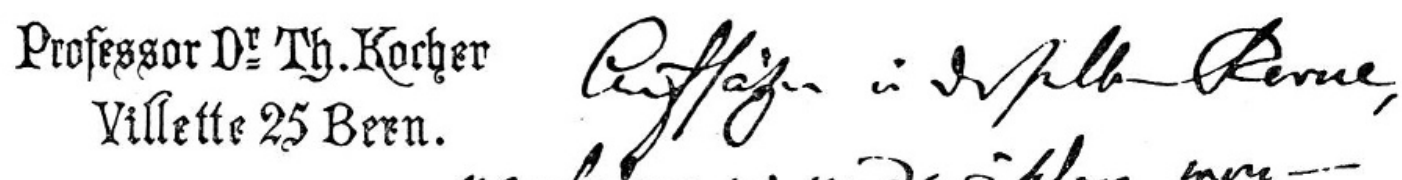

nafe w...jorifun mon-..

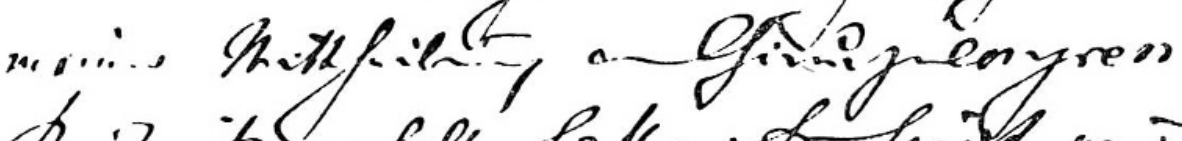

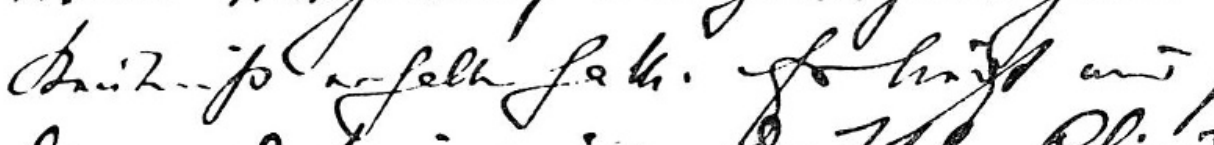

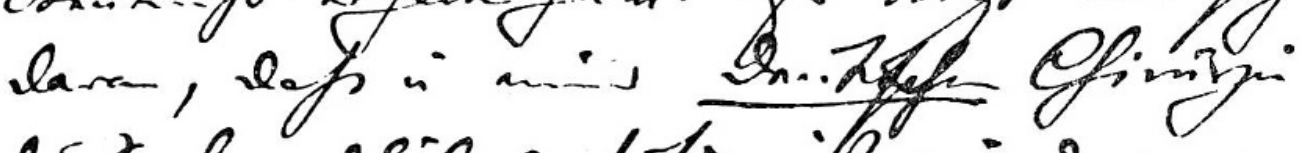

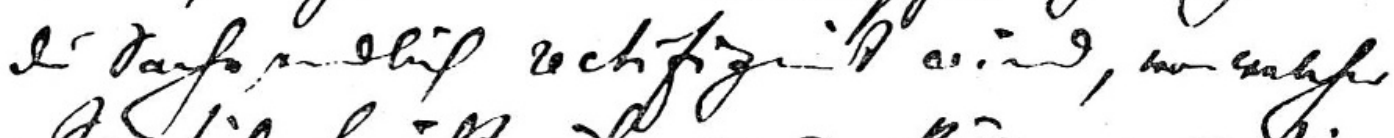

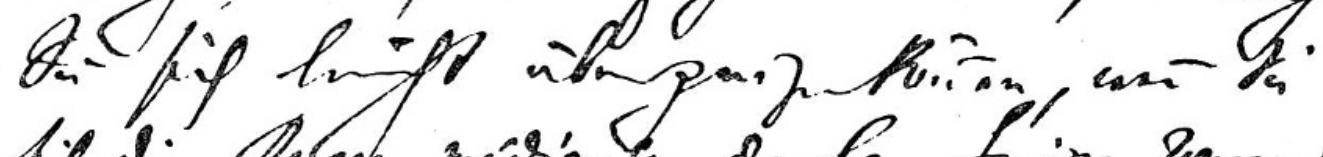

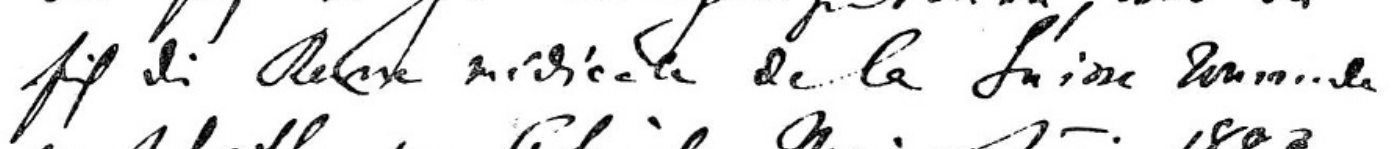

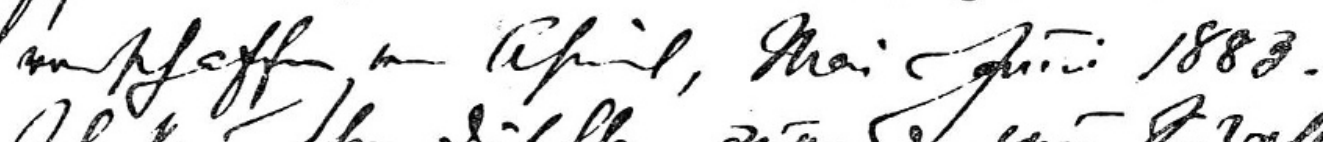

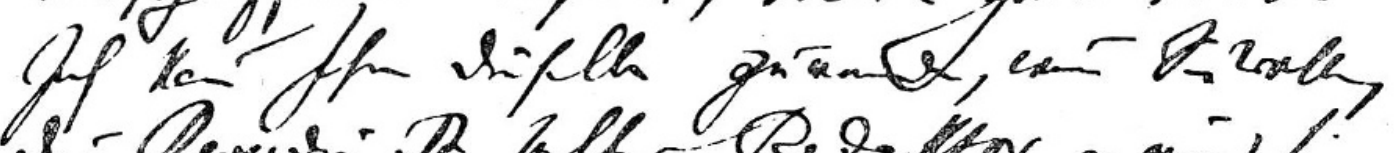

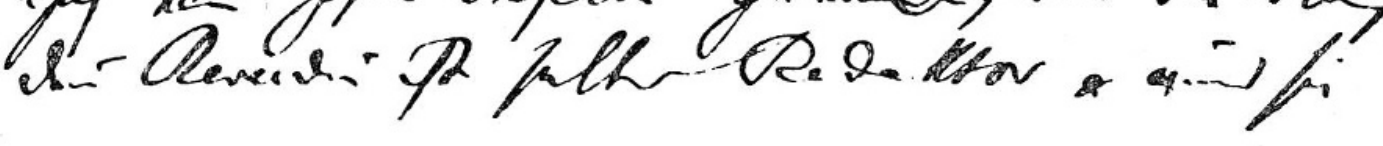

Zweite Karte - Vorderseite

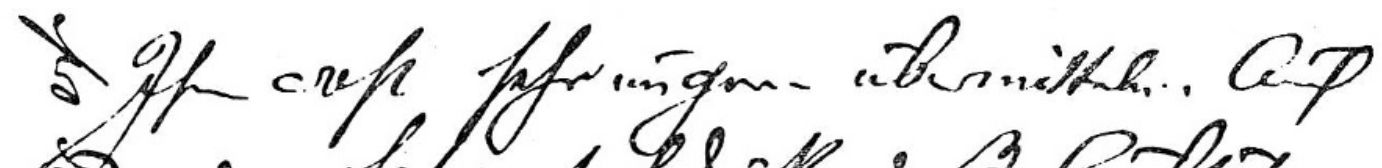

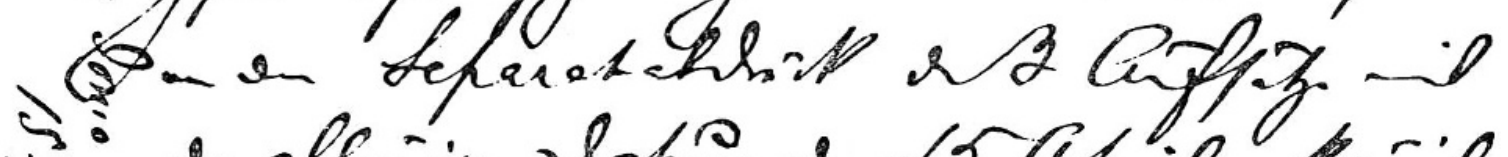

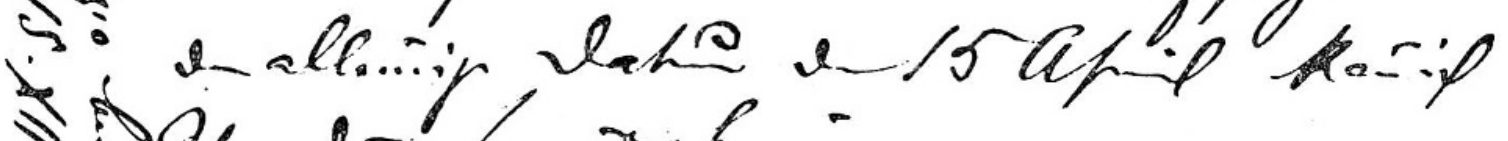

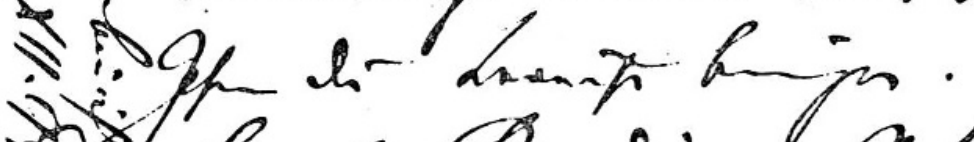

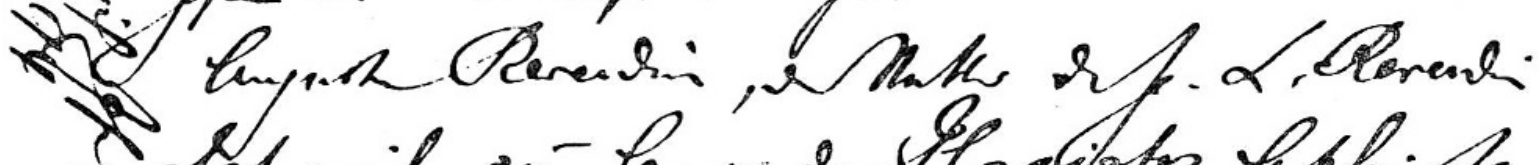

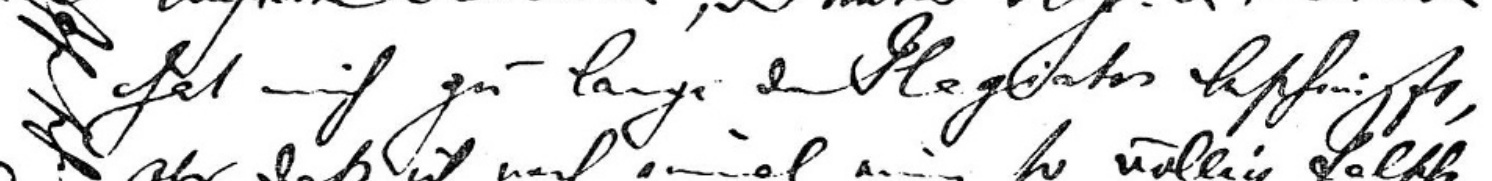

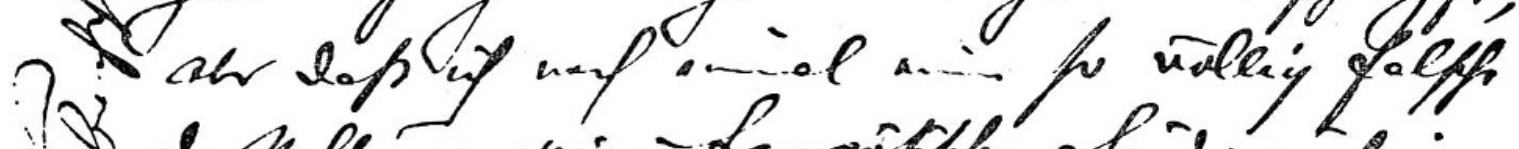

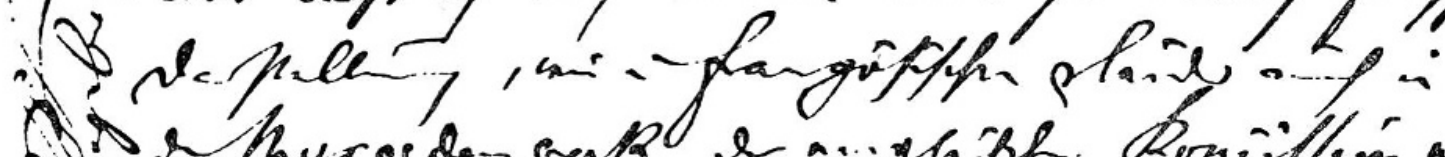

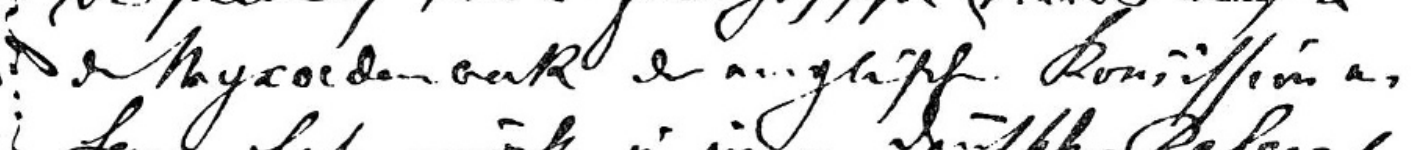

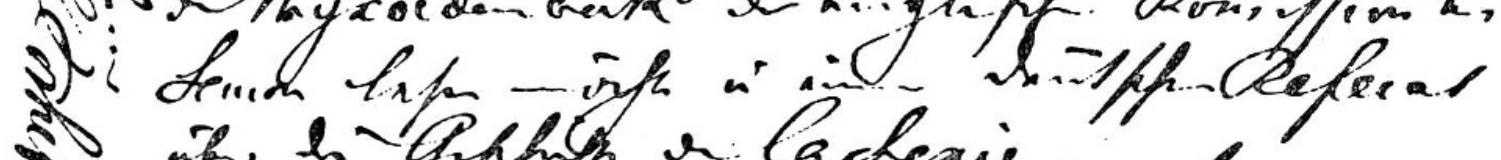

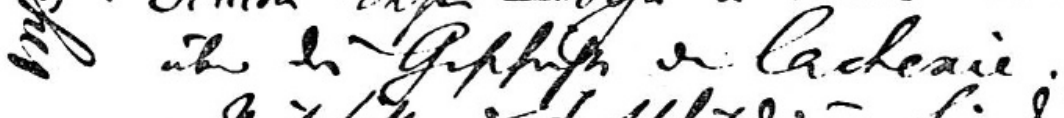

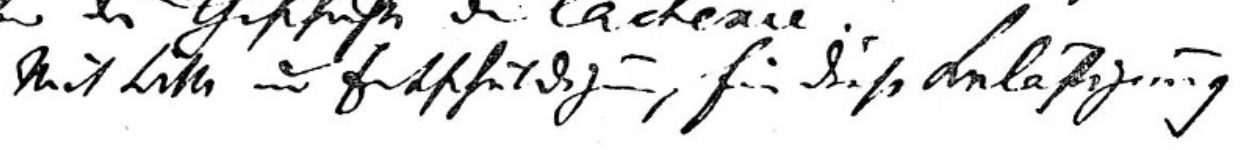

Zweite Karte - Rückseite 


\section{Die Briefe Th. Kochers}

\section{Mein lieber Herr Kollege!}

Da ich erfahren habe, daß Sie für die Deutsche Chirurgie die Kröpfe, recte Krankheiten der Schilddrüse zu bearbeiten übernommen haben, so bin ich so frei, Sie zu bitten, in der Frage der «Erfindung» der Cachexia strumipriva Ihren klaren Standpunkt zu nehmen[.] Zu diesem Behuf sende ich Ihnen die Brochüre von Dr. Lardy zu, in welcher der Nachweis geleistet ist, wie wenig vor mir Prof. Reverdin in Genf eine Ahnung hatte, von einem constanten causalen Zusammenhang zwischen Excision der Schilddrüse und der Cachexie. Die Franzosen haben einfach die Reverdin'schen Ansprüche, weil er ein Mitglied ihrer Gesellschaft ist, zu den ihrigen gemacht und die Aufnahme einer Rectifikation in die Revue de chirurgie gegenüber den lügnerischen Ansprüchen des H (errn) Auguste Reverdin rundweg verweigert. Wie Reverdin selbst bei den Engländern den Schein einer Priorität erwecken konnte, so geschah dieß bloß deßhalb, weil er das Datum fälschte, d.h. seine in den Monaten April, Mai und Juni 1883 erschienenen Aufsätze (in der Revue méd. de la Suisse romande) in einem Separatabzug unter dem Datum bloß des 15 April versandte. Und Sie werden doch sofort sehen, welch' ein gewaltiger Unterschied besteht zwischen der Veröffentlichung des ersten Aufsatzes im April und den späteren Aufsätzen in derselben Revue, nachdem er inzwischen von meiner Mittheilung am Chirurgencongreß Kenntniß erhalten hatte. Es liegt mir sehr daran, daß in einer deutschen Chirurgie die Sache endlich rectifiziert wird, von welcher Sie sich leicht überzeugen können, wenn Sie sich die Revue médicale de la Suisse Romande verschaffen vom April, Mai und Juni 1883. Ich kann Ihnen dieselbe zusenden, wenn Sie wollen, denn Reverdin ist selber Redaktor und wird sie Ihnen wohl sehr ungern übermitteln. Auch von dem Separatabdruck der 3 Aufsätze mit dem alleinigen Datum des 15 April kann ich Thnen die Beweise bringen.

Auguste Reverdin, der Vetter des J.L. Reverdin hat mich zu lange des Plagiates beschimpft, als daß ich noch einmal eine so völlig falsche Darstellung, wie sie im französischen und leider auch in dem Myxoedemwerk der englischen Kommission von Semon lesen möchte in einem deutschen Referat über die Geschichte der Cachexie.

Mit Bitte um Entschuldigung für diese Belästigung pro domo bin ich Ihr sehr ergebener

15.XII.94

Th. Kocher 


\section{Mein lieber Herr Kollege!}

Es ist mir leid, Sie zu plagen, aber ich halte doch darauf, daß Sie bei Ihrer Darstellung der Geschichte der Cachexia thyreopriva sich selber überzeugen, daß meine Ansprüche auf Priorität gerechtfertigt sind und sende Ihnen daher die bdn. Brochüren zu, um deren gütige Rücksendung ich bitte, wenn Sie Zeit gehabt haben, sich mit der Sache zu befassen. Sie sehen aus der Einlage, daß auch Ewald, der darüber zu schreiben hat, sich überzeugt hat, auf wessen Seite das Recht ist und wenn Sie die Brochüre von Dr. Lardy lesen und den Vergleich ziehen zwischen dem, was in den beiden Heften vom April und Mai der Revue médicale steht - welche vor Kenntnißnahme meiner Mittheilung am Chirurgencongreß gedruckt sind - und dem was das Juniheft enthält, so werden Sie sehen, daß Reverdin, als er meine Mittheilung gelesen hatte, seine Patienten hat wieder kommen lassen und nun in vollkommen anderer Weise beurtheilt und beschrieben hat.

Sie begreifen auch, warum er in dem Separatabdruck, den ich beilege, die Daten von Mai und Juni wegließ und bloß das Aprildatum hinsetzte und daß dieses Verfahren geeignet war, die Leute zu täuschen, welche die Arbeit nur in ihrem letzten interessanteren Theil studierten und die Revue médicale selbst nicht kannten, so Felix Semon und das Myxoedemkomité in London.

Sie werden auch aus den glänzenden Resultaten der Totalexcision, die noch im April und Mai so unbefangen publiziert wurden, ersehen können, wie sehr die erste kurze Notiz Reverdins im Herbst 1882 von dem Gespräch beeinflußt war, das wir 8 Tage vorher am internat. Hygiène-Congreß zusammen gehabt haben.

Mit freundlichem Gruß!

Ihr ergebener

Th. Kocher

Ich hoffe, Ihre Ferien haben Sie und Ihre geehrte Frau Gemahlin recht erquickt und lassen Sie die Winterarbeit mit rechter Frische wieder aufnehmen.

Der Inhalt der Briefe dürfte weitgehend jenes Urteil bestätigen, zu dem Bornhauser in seiner historischen Untersuchung gelangte, er dürfte freilich auch die Haltung der beiden Kontrahenten in einem noch deutlicheren 
Licht erscheinen lassen. Als interessante Einzelheit mag dabei vermerkt werden, daß Jacques Louis Reverdin bereits in seinem ersten Brief aus dem Jahre 1889 ausdrücklich darauf hinweist, die gemeinsam mit Vetter Auguste verfaßte Kasuistik ${ }^{16}$ sei in den Nummern April, Mai und Juni der Révue Médicale de la Suisse Romande erschienen. Er entkräftet damit im voraus jene Unterstellungen, die Kocher in seinem Schreiben an Combe ${ }^{17}$ und noch sehr viel eindeutiger in den beiden vorliegenden Briefen ausgesprochen hat. Zudem tat dieser dem Genfer Chirurgen bitter Unrecht, wenn er glaubte, Reverdin werde die Arbeit nur sehr ungern seinem Wiener Kollegen übermitteln.

Auch der zweite Brief wehrt sich nur gegen jene Anwürfe ${ }^{18}$, die bereits Bornhauser als unberechtigt erkannt hat. Wie Combe ${ }^{19}$, so versichert er auch v. Eiselsberg, Kocher habe in ihrem gemeinsamen Gespräch anläßlich des Hygienekongresses am 7.September 1882 auf seine (Reverdins) Frage, ob auch er (Kocher) Ausfallserscheinungen nach Totalexstirpation der Schilddrüse gesehen habe, zunächst mit «Nein » geantwortet. Und indem er sein Schreiben an den Berner Chirurgen vom 21. Mai des Jahres 1883 erwähnt, bestätigt auch er, daß zwischen den beiden Gelehrten in dieser Auseinandersetzung Briefe gewechselt worden sind. Neu und bemerkenswert ist am Ende freilich die Tatsache, daß sich Jacques Louis Reverdin von den Ansprüchen seines Vetters Auguste ${ }^{20}$, die auch Bornhauser als übertrieben ablehnt ${ }^{21}$, expressis verbis distanziert hat. Die letzten Sätze seines zweiten Briefes zeigen noch einmal deutlich, daß Reverdin maßvoll und ohne jede unnötige Polemik nur jene Verdienste verteidigte, die ihm später auch die historische Forschung zubilligen mußte.

Schon Bornhauser hat auf die ungewöhnliche Schärfe und die eigentümlichen Unterstellungen hingewiesen, mit denen dieser Streit von Kochers Seite geführt wurde ${ }^{22}$, und beide Briefe bestätigen dieses Vorgehen in fast bestürzender Weise, ohne da $\beta$ auf die Einzelheiten noch einmal näher eingegangen zu werden braucht,. Sie zeigen, da $\beta$ Reverdin in der Tat darauf Wert legen mußte, wie er am Ende seines zweiten Briefes schreibt, einen

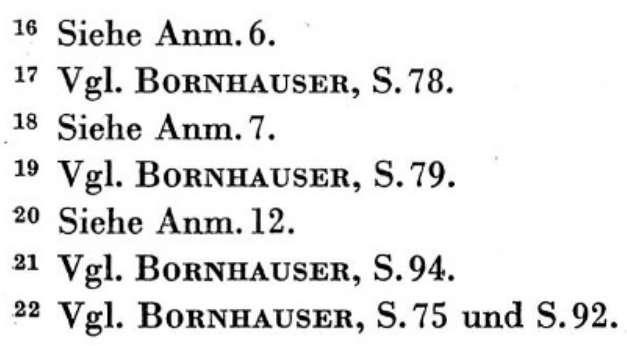


Angriff auf seine wissenschaftliche Ehre abzuwehren. Erst die Kenntnis dieser beiden Briefe läßt es verständlich erscheinen, daß Adolphe Combe Kochers Schreiben nicht vollständig publizierte, sondern das überging, «qui m'était personnel $\gg{ }^{23}$. Dabei versteift sich Kocher vor allem auf das Argument, der entscheidende Teil der «Note sur vingt-deux opérations de goitre» sei erst im Juni-Heft publiziert, und dieses Datum nachträglich bewußt verschleiert worden. Gerade dieser Vorwurf aber dürfte nun mit der Kenntnis von Reverdins erstem Brief endgültig gegenstandslos geworden sein.

Über das Gespräch auf dem internationalen Hygienekongreß hat sich Kocher allerdings sehr viel vorsichtiger geäußert, als es nach den bisher bekannten Publikationen den Anschein haben mochte, und so erscheint es doch zunächst überraschend, wenn v.Eiselsberg in seiner Darstellung schreibt: «Er (Kocher) giebt zu, daß Reverdin zuerst im September 1882 einige Symptome der Kachexie publicirt habe, aber eben 6 Tage nach der Unterredung mit Kocher, wobei er (Kocher) Reverdin gesagt haben soll, daß der von Kocher im Jahre 1874 operirte Patient einem dem Cretinismus ähnlichen Marasmus verfallen war, und er (Kocher) sich mit der Untersuchung dieser Fälle beschäftige. ${ }^{24}$ Auf dieser Darstellung fußt auch noch Bornhausers Untersuchung ${ }^{25}$, doch dürfte Kochers kurze Bemerkung am Ende seines zweiten Briefes eine solche Interpretation kaum zulassen. Hier mag der Wiener Chirurg auf die Arbeit von Lardy gebaut haben, wenn man nicht ein drittes Schreiben Kochers annehmen will, das verloren ist.

v. Eiselsbergs Stellungnahme gelangt als Resultat dieser Briefe zu einem Urteil, das mit den Ergebnissen Bornhausers bereits weitgehend übereinstimmt, und er war es auch, der später für die Anerkennung Reverdins als Erstentdecker eingetreten ist ${ }^{26}$. Andererseits muß es verständlich erscheinen, wenn er die Streitfrage in seinen Publikationen aus einem gewissen Abstand betrachtete. Denn in seinen Lebenserinnerungen hat er später im Kapitel über seine eigenen "Versäumten Gelegenheiten» geäußert: «Für die praktische Chirurgie ist es völlig gleichgültig, wer der Vater der neuen Vorschläge war, die Hauptsache ist, daß sie gemacht wurden und daß die Methoden den Kranken nützen ${ }^{27}$. So steht neben seinem Streben nach einer

23 Vgl. Bornhauser, S. 78.

24 Vgl. A. Frhr. v. Eiselsberg, Die Krankheiten der Schilddrüse, S. 150 (Anm. 1 von S. 149); zitiert bei BorNHAUSER, S. 81.

25 Vgl. Bornhauser, S. 95.

26 Vgl. BORNhauser, S. $111 \mathrm{ff}$.

27 A. Frhr. v. Eiselsberg, Lebensweg eines Chirurgen, S. 550. 
gerechten Würdigung unverkennbar der wohlmeinende Versuch, beschwichtigend und ausgleichend zu wirken. Ein solches Bemühen scheint ihn sogar bestimmt zu haben, die Verdienste der Billroth-Schule auf dem Gebiet der Schilddrüsenforschung und der Kropfchirurgie - und damit nicht zuletzt seine eigenen - ein wenig in den Hintergrund zu rücken ${ }^{28}$. Besonders deutlich kommt es in jenen Sätzen seiner Monographie zum Ausdruck, in denen er die Leistung beider anerkennt: "Wir müssen beiden Chirurgen für ihre Forschungen dankbar sein. Durch ihre Beobachtungen haben sie den kropf kranken Menschen einen unschätzbaren Dienst erwiesen, da sonst wohl noch viel mehr Unheil durch die momentan so vortrefflich wirkende Totalexstirpation angerichtet worden wäre » ${ }^{29}$. Auch in späteren Arbeiten hat er stets beide Chirurgen gemeinsam und Reverdin an erster Stelle genannt ${ }^{30}$. Einem Mann, der so dachte, muß die Hartnäckigkeit einer solchen Auseinandersetzung im Grunde fremd geblieben sein. Kocher aber vermochte nicht mehr nachzugeben, und auch in der von ihm edierten «Encyklopädie der gesamten Chirurgie» wird in dem von seinem Sohn Albert verfaßten Artikel über die operative Behandlung der Struma Reverdin mit Stillschweigen übergangen ${ }^{31}$.

28 Vgl.E.LESKy, Die Wiener Medizinische Schule, Graz/Köln 1965, S.394 und S.443.

29 Vgl. A. Frhr. v. Eiselsberg, Die Krankheiten der Schilddrüse, S.150.

30 Vgl. A.Frhr. v.EISELSBerg, Der Werth der Schilddrüse im Haushalte der Natur, Verh. Ges. Dtsch. Naturforscher und Ärzte, 74. Versammlung (1902) Teil 1, Leipzig 1903, S. 65 und ders., Die Chirurgie der Schilddrüse, in: Handbuch der praktischen Chirurgie, Band II, Stuttgart 1913, S.391f.

31 A. Kocher, Art. Struma, operative Behandlung, in: Encyklopädie der gesamten Chirurgie, herausgegeben von Th. Kocher und F. de Quervarn, Band II, Leipzig 1903, S.504. 